(2) Open Access Full Text Article

\title{
A case of developing progressive multifocal leukoencephalopathy while using rituximab and mycophenolate mofetil in refractory systemic lupus erythematosus
}

This article was published in the following Dove Press journal:

Therapeutics and Clinical Risk Management

\section{Yuichi Ishikawa \\ Tadamichi Kasuya \\ Junichi Ishikawa \\ Michio Fujiwara \\ Yasuhiko Kita}

Department of Rheumatology, Yokohama Rosai Hospital, Kohoku-ku, Yokohama, Kanagawa, Japan
Correspondence: Yasuhiko Kita

Department of Rheumatology, Yokohama Rosai Hospital, 32II Kozukue-cho,

Kohoku-ku, Yokohama,

Kanagawa 222-0036, Japan

Tel $+8 \mathrm{I} 454748 \mathrm{II}$ I

Fax +8I 454748323

Email kitayas@yokohamah.johas.go.jp

\begin{abstract}
Progressive multifocal leukoencephalopathy (PML) is a central nervous system infection caused by John Cunningham (JC) virus reactivation in an immunocompromised patient. PML has various neurologic symptoms and has very poor prognosis. A 36-year-old man developed transverse myelitis and had a psychiatric disorder at the age of 26 . He was diagnosed with systemic lupus erythematosus (SLE) and neuropsychiatric SLE (NPSLE), on the basis of leukopenia and presence of anti-DNA and anti-nuclear antibodies. Treatment with glucocorticoid (GC) was started, and remission was introduced. Six months before PML onset, his condition was complicated with hemophagocytic lymphohistiocytosis (HLH) due to exacerbation of SLE. Remission re-induction therapy by GC, cyclosporine-A, intravenous cyclophosphamide, and rituximab (RTX) was initiated and HLH improved. However, interleukin-6 levels of the cerebrospinal fluid (CSF) continued to rise. We thought that the disease activity of NPSLE worsened; thus, we introduced mycophenolate mofetil (MMF) 4 months before the PML onset. He developed progressive dysarthria and right hemiplegia. He was diagnosed with PML via magnetic resonance imaging and JC virus polymerase chain reaction in CSF. Considering that immunosuppressants, including RTX and MMF, are precipitating factors of PML, we discussed the RTX removal using plasma exchange (PEx), but we did not introduce PEx, because it was expected that the concentration of RTX was already lowered when he was diagnosed with PML. Treatment for PML with mefloquine and mirtazapine saved his life, but severe residual disabilities remained. This is the first report of a patient who developed PML during combination therapy with RTX and MMF.
\end{abstract}

Keywords: progressive multifocal leukoencephalopathy, systemic lupus erythematosus, rituximab, mycophenolate mofetil, mefloquine

\section{Introduction}

Progressive multifocal leukoencephalopathy (PML) is a demyelinating disease of the central nervous system caused by reactivation of John Cunningham (JC) virus in an immunocompromised patient. PML has various neurological symptoms, such as paralysis or dysphagia. Previously, PML was mostly seen in patients with HIV/AIDS. Along with the advancement of immunosuppressive therapy for various autoimmune diseases, PML based on autoimmune diseases is increasing. ${ }^{1}$ With the advent of new immunosuppressive agents, the use of combination therapy may also increase. Therefore, there is a possibility that severe complications including PML will increase. In some non-HIV associated PML cases, mirtazapine, mefloquine, cytarabine, and 
cidofovir have been effective. ${ }^{2,3}$ In particular, mefloquine, an antimalarial drug, has been reported to inhibit the proliferation of JC virus in vitro and is often used for the treatment of non-HIV PML. ${ }^{4}$ However, non-HIV PML has a very poor prognosis. The therapeutic strategy for non-HIV PML has not been established. Here, we report a patient who developed PML during the combination therapy with rituximab (RTX) and mycophenolate mofetil (MMF) in refractory systemic lupus erythematosus (SLE) and the valuable effect of mefloquine and mirtazapine in PML.

\section{Case report}

The patient was a 36-year-old Japanese man. He developed transverse myelitis and psychiatric disorder at 26 years of age. He was diagnosed as having SLE and neuropsychiatric SLE (NPSLE) based on the presence of leukopenia and positive anti-DNA and anti-nuclear antibodies. Treatment with glucocorticoid (GC) was initiated, and remission was introduced. Six months before the PML onset, his condition was complicated with hemophagocytic lymphohistiocytosis (HLH), and SLE relapsed. Remission reinduction therapy, consisting of two courses of GC pulse therapy (methylprednisolone at 1,000 mg/day), cyclosporine-A, intravenous cyclophosphamide (IVCY), and RTX, was administered. One course of IVCY at $500 \mathrm{mg}$ was administered. However, IVCY was ineffective for HLH. We administered four courses of RTX at $375 \mathrm{mg} / \mathrm{m}^{2}$. The final administration of RTX was 5 months before PML onset (Figure 1). After remission reinduction therapy, HLH was improved. GC was gradually tapered.
However, interleukin-6 (IL-6) levels in the cerebrospinal fluid (CSF) were gradually rising (maximum IL-6 level recorded just before the start of MMF administration was $16.4 \mathrm{pg} / \mathrm{mL}$ ). We diagnosed that the disease activity of NPSLE deteriorated. Because we thought that therapy intensification for NPSLE was required, we introduced MMF 4 months before PML onset. MMF was started at 1,000 mg/day and was gradually increased to $3,000 \mathrm{mg} / \mathrm{day}$, and GC was gradually tapered. Four months after starting MMF, he developed dysarthria and right hemiplegia. Brain magnetic resonance imaging (MRI) showed an abnormal focal lesion in the white matter of the temporal lobe (left lobe dominant) (Figure 2A). White matter lesions gradually extended (Figure 2B). Polymerase chain reaction (PCR) tests for JC virus were positive in the CSF (96,935,000 copies/ $\mu \mathrm{L})$. Finally, the patient was diagnosed with PML 3 months after the onset of dysarthria and right hemiplegia. Considering that immunosuppressive therapy is a precipitating factor of PML, RTX may have affected the onset of PML. Thus, we considered RTX removal using plasma exchange (PEx), but we did not introduce PEx, because it was expected that the concentration of RTX was already lowered at the time he was diagnosed with PML. Simultaneously with the diagnosis of PML, we started to taper immunosuppressive agents gradually so that the disease activity of SLE does not recur (GC gradually decreased from prednisolone 8 to $5 \mathrm{mg}$ /day and MMF was tapered off). In addition, after obtaining the approval of our hospital's ethics committee, we started to administer mefloquine and mirtazapine, which were reported to have therapeutic effects for non-HIV

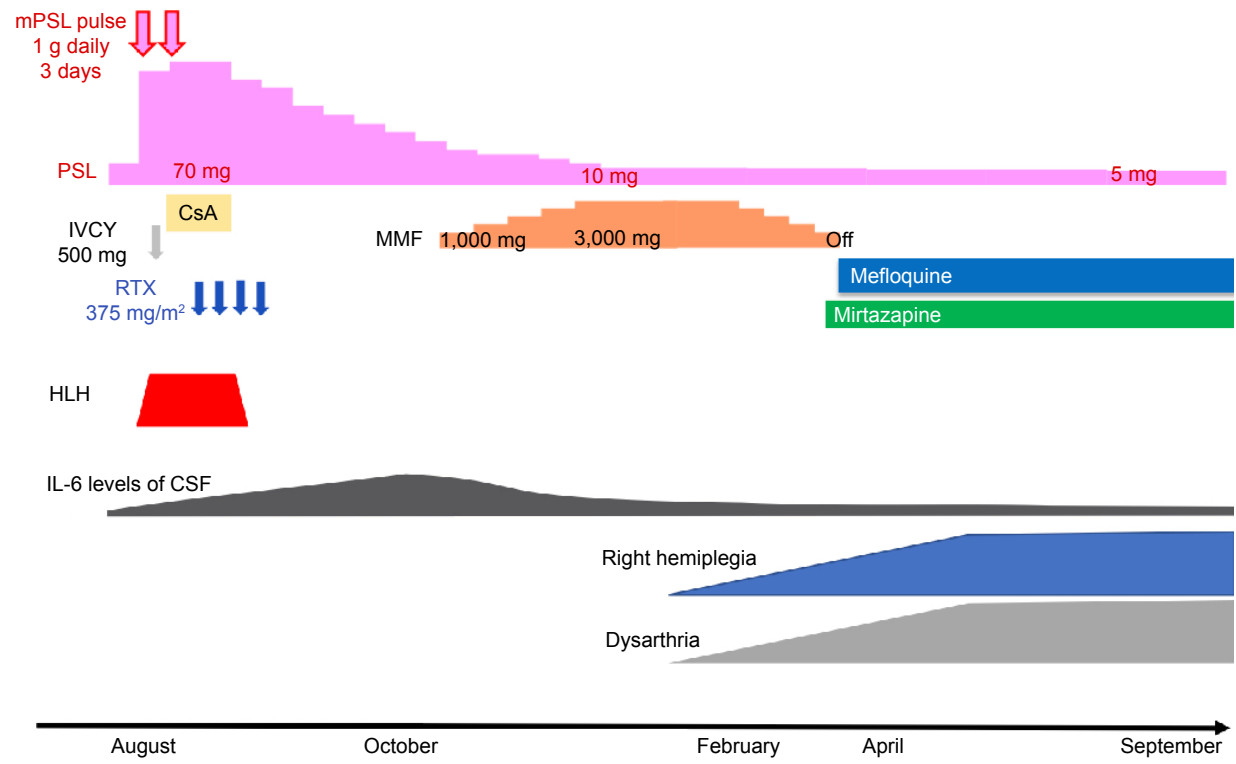

Figure I The clinical course and treatments.

Abbreviations: mPSL, methylprednisolone; PSL, prednisolone; CsA, cyclosporine-A; IVCY, intravenous cyclophosphamide; RTX, rituximab; MMF, mycophenolate mofetil; $\mathrm{HLH}$, hemophagocytic lymphohistiocytosis; IL-6, interleukin-6; CSF, cerebrospinal fluid. 

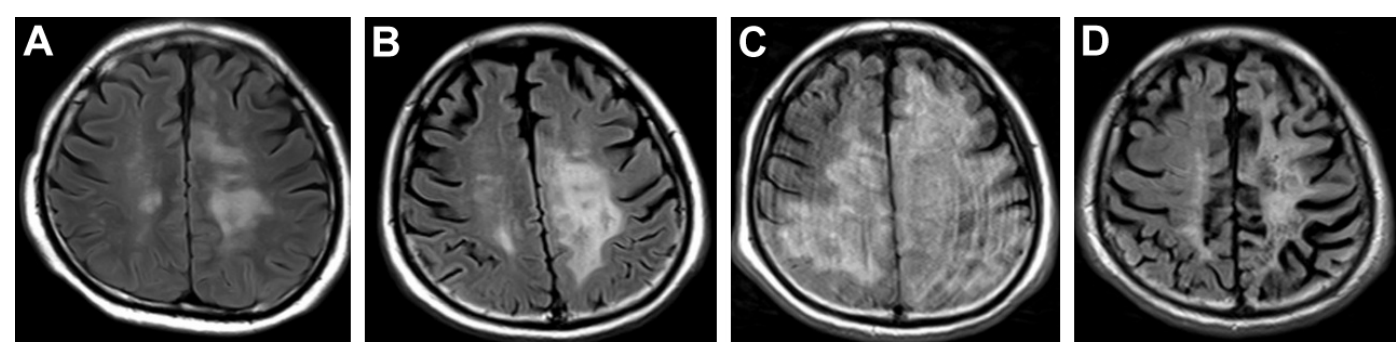

Figure 2 Changes in brain magnetic resonance imaging (FLAIR images).

Notes: (A) At the time of the PML onset. (B) A month and half after PML onset before starting treatment by mefloquine and mirtazapine. (C) Two months after the start of treatment by mefloquine and mirtazapine. (D) Eight months after the onset of PML and 6 months after the start of treatment by mefloquine and mirtazapine.

Abbreviations: FLAIR, fluid-attenuated inversion recovery; PML, progressive multifocal leukoencephalopathy.

associated PML., ${ }^{3,5}$ One month after starting mefloquine and mirtazapine administration, spiking fever, and serological inflammatory response were observed, without obvious signs of infection. Although immune reconstitution syndrome (IRIS) was suspected, the patient did not have vital organ complications. Therefore, we did not increase GC for IRIS. Fever and rise of serological inflammatory response lasted $\sim 2$ weeks, his fever and serological inflammatory response declined spontaneously without increase of GC. Two months after starting mefloquine and mirtazapine administration, white matter lesions further extended (Figure 2C). The patient began to suffer life-threatening epileptic seizures and needed anticonvulsants. Six months after starting treatment with mefloquine and mirtazapine, epileptic seizures became controllable with anticonvulsants, thus saving his life. Although MRI findings revealed improvement of white matter lesions in the brain and the load of JC virus DNA in the CSF was drastically decreased ( 7,492 copies $/ \mu \mathrm{L})$, extensive atrophy of the brain parenchyma was observed (Figure 2D). Although his activities of daily living were independent before the onset of PML, consequentially, he had severe physical impediment (dysarthria and hemiplegia). He needed total assistance in doing his activities of daily living. The patient had a functional independence measure score of $19 .{ }^{19}$ Even though immunosuppressive therapy was reduced, there was no subsequent recurrence of SLE or NPSLE 11 months after tapering of immunosuppressive therapy and initiation of mefloquine and mirtazapine.

Written informed consent in Japanese was provided by the patient's father to have the case details and accompanying images published.

\section{Discussion}

Cellular immune deficiency or other kinds of immunosuppression (eg, immunosuppressive therapy and anticancer drug treatment) allow the reactivation of JC virus. It is thought that PML develops through the reactivation of JC virus, infecting and destroying oligodendrocytes, possibly through the $5-\mathrm{HT}_{2 \mathrm{~A}}$ serotonin receptor. ${ }^{6}$ In the past, most PML cases occurred in patients with HIV/AIDS. In recent years, reports of PML occurrence during immunosuppressive therapy are increasing with the rise of various immunosuppressive and biological agents, such as RTX for various autoimmune diseases. ${ }^{7}$ Patients using immunosuppressive agents other than biologic agents, such as GC, MMF, cyclosporine-A, and IVCY, are also reported to be at risk of developing PML. Immunosuppressive agents used at the onset of PML varied, and the timing of onset was also diverse. ${ }^{7}$ Therefore, it is difficult to accurately predict the onset time of PML. Among the connective tissue diseases, the incidence of PML in SLE is relatively high. ${ }^{8}$ A retrospective large population-based observational study, which analyzed a health insurance database, showed that the incidence rate of PML in patients with SLE is 2.4 per 100,000 person-years. ${ }^{9}$ Nearly two-thirds of PML cases in patients with systemic rheumatic diseases occurred in patients with SLE as reported in the literature. ${ }^{8}$ Forty percent of PML cases in SLE occurred in patients who had had minimal immunosuppression (ie, low-dose GC therapy without other immunosuppressive agents). ${ }^{10}$ It is notable that only the maintenance doses of GC or immunosuppressive agents could have developed PML. This suggested that SLE itself may predispose to PML. In our case, we were forced to administer GC, RTX, and MMF simultaneously for refractory SLE and NPSLE, and temporarily used concomitantly more immunosuppressive agents. Since multiple immunosuppressive agents were administered, our case is considered to be a high-risk case of PML. This combination therapy of RTX and MMF has been reported to be effective for refractory SLE with various organ complications and childhood GCresistant nephrotic syndrome. ${ }^{11,12}$ In each report, although infectious diseases were reported as adverse events, there was no mention that adverse events including infections had increased compared with conventional therapies. Moreover, 
there were no cases of developing PML. On the other hand, it is suggested that both RTX and MMF may be risk factors for developing PML. Henegar et al reported that two out of 35 patients with SLE who developed PML had a history of RTX administration and nine of the patients had history of MMF administration. ${ }^{13}$ However, there is no report that PML occurred during combination therapy with RTX and MMF. This is the first report on PML that occurred during the combination therapy with RTX and MMF in a patient with SLE. It is necessary to pay attention to the onset of PML regardless of the therapeutic regimen in SLE patients. In our case, the final administration of RTX was 5 months before PML onset. The administration of RTX results in depletion of $\mathrm{CD} 20^{+} \mathrm{B}$ cells in the peripheral blood and CSF. ${ }^{14}$ Subsequently, recruitment of immature B cells from the bone marrow potentially disseminates the latent virus to the brain. RTX attenuates immune surveillance systems. ${ }^{15}$ Therefore, it is thought that the effect of RTX remained, and RTX affected the onset of PML. In non-HIV PML, discontinuation or tapering of immunosuppressive therapy is the primary therapy for PML. Additionally, several studies have reported that mefloquine alone or in combination with mirtazapine was effective for non-HIV PML. ${ }^{3,5}$ Mefloquine has been shown to have anti-JC virus action in vitro, and mirtazapine is supposed to inhibit JC virus from invading oligodendroglia. ${ }^{3,4}$ In this case, it seems that treatment with mefloquine and mirtazapine was considered partially successful because the JC virus DNA levels in the CSF were drastically decreased. However, the recent mefloquine trial of PML patients was reported to fail to reduce JC virus DNA levels in the CSF. ${ }^{16}$ In this trial, a total of 37 PML patients were registered and randomized to the standard of care group or mefloquine group. This included $21 \mathrm{HIV}$-positive patients, all of whom were taking highly active antiretroviral therapy (HAART), and three HIV-negative patients who had either cancer or systemic rheumatic disease. The background condition, status of HIV with HAART or non-HIV, might have affected the results. It has been reported that cytarabine and cidofovir were effective for non-HIV PML, but the effectiveness was not demonstrated by analysis of a large number of subjects. ${ }^{2}$ Thus, no clear evidence has been established for the treatment of non-HIV PML. Further studies in a large number of subjects should be conducted to confirm whether mefloquine is effective for treading non-HIV PML. When treating PML, we should pay attention to IRIS. Cellular immunity is ameliorated by tapering of immunosuppressive therapy, attacking JC virus-infected cells, which triggers a rapid inflammatory reaction and development of IRIS.
The immune reaction after immune reconstitution is a vital response necessary to remove JC virus-infected cells; for mild cases like the presented case, only follow-up observation is acceptable. However, in some severe cases, such as a vital organ involvement, treatment of IRIS with GC is needed, because there is a risk of death or severe disability. ${ }^{17} \mathrm{In}$ some of these cases, as well as the present case, the patients showed decreased JC virus DNA levels in the CSF. ${ }^{18}$ Therefore, JC virus DNA levels in the CSF may be an indicator of therapeutic effect. Although treatment with mefloquine and mirtazapine saved our patient's life, it is very disappointing that severe physical impediments remained. It is conceivable that the risk of PML onset could be increased due to the combination therapy with RTX and MMF.

\section{Conclusion}

It was concluded that careful attention should be paid to the onset of PML when using the combination therapy with RTX and MMF, especially in patients with SLE. When PML develops, it may be worth trying treatment with mefloquine and mirtazapine.

\section{Disclosure}

The authors report no conflicts of interest in this work.

\section{References}

1. Chahin S, Berger JR. A risk classification for immunosuppressive treatment-associated progressive multifocal leukoencephalopathy. J Neurovirol. 2015;21(6):623-631.

2. Terrier B, Hummel A, Fakhouri F, et al. Leucoencéphalite multifocale progressive en dehors du sida : efficacité de l'association cytarabinecidfovir. [Progressive multifocal leukoencephalopathy in a non-AIDS patient: high efficiency of combined cytarabine and cidofovir]. Rev Med Interne. 2007;28(7):488-491. French.

3. Epperla N, Medina-Flores R, Mazza JJ, Yale SH. Mirtazapine and mefloquine therapy for non-AIDS-related progressive multifocal leukoencephalopathy. WMJ. 2014;113(6):242-245.

4. Brickelmaier M, Lugovskoy A, Kartikeyan R, et al. Identification and characterization of mefloquine efficacy against JC virus in vitro. Antimicrob Agents Chemother. 2009;53(5):1840-1849.

5. Beppu M, Kawamoto M, Nukuzuma S, Kohara N. Mefloquine improved progressive multifocal leukoencephalopathy in a patient with systemic lupus erythematosus. Intern Med. 2012;51(10):1245-1247.

6. Elphick GF, Querbes W, Jordan JA, et al. The human polyomavirus, JCV, uses serotonin receptors to infect cells. Science. 2004;306(5700): 1380-1383.

7. Molloy ES, Calabrese LH. Progressive multifocal leukoencephalopathy associated with immunosuppressive therapy in rheumatic diseases: evolving role of biologic therapies. Arthritis Rheum. 2012;64(9): 3043-3051.

8. Molloy ES, Calabrese LH. Progressive multifocal leukoencephalopathy in patients with rheumatic diseases: are patients with systemic lupus erythematosus at particular risk? Autoimmun Rev. 2008;8(2):144-146.

9. Amend KL, Turnbull B, Foskett N, et al. Incidence of progressive multifocal leukoencephalopathy in patients without HIV. Neurology. 2010; 75(15):1326-1332. 
10. Palazzo E, Yahia SA. Progressive multifocal leukoencephalopathy in autoimmune diseases. Joint Bone Spine. 2012;79(4):351-355.

11. Galarza-Maldonado C, Kourilovitch MR, Molineros JE, et al. The administration of low doses of rituximab followed by hydroxychloroquine, prednisone and low doses of mycophenolate mofetil is an effective therapy in Latin American patients with active systemic lupus erythematosus. Autoimmun Rev. 2010;10(2):108-111.

12. Basu B, Mahapatra TK, Mondal N. Mycophenolate mofetil following rituximab in children with steroid-resistant nephrotic syndrome. Pediatrics. 2015;136(1):132-139.

13. Henegar CE, Eudy AM, Kharat V, et al. Progressive multifocal leukoencephalopathy in patients with systemic lupus erythematosus: a systematic literature review. Lupus. 2016;25(6):617-626.

14. Major EO. Progressive multifocal leukoencephalopathy in patients on immunomodulatory therapies. Annu Rev Med. 2010;61:35-47.
15. Bellizzi A, Anzivino E, Rodio DM, et al. New insights on human polyomavirus JC and pathogenesis of progressive multifocal leukoencephalopathy. Clin Dev Immunol. 2013;2013:839719.

16. Clifford D, Nath A, Cinque P, et al. A study of mefloquine treatment for progressive multifocal leukoencephalopathy: results and exploration of predictors of PML outcomes. J Neurovirol. 2013;19(4):351-358.

17. Elston JW, Thaker H. Immune reconstitution inflammatory syndrome. Int J STD AIDS. 2009;20(4):221-224.

18. Bossolasco S, Calori G, Moretti F, et al. Prognostic significance of JC virus DNA levels in cerebrospinal fluid of patients with HIV-associated progressive multifocal leukoencephalopathy. Clin Infect Dis. 2005;40(5):738-744.

19. Keith RA, Granger CV, Hamilton BB, Sherwin FS. The functional independence measure: a new tool for rehabilitation. Adv Clin Rehabil. 1987;1:6-18.
Therapeutics and Clinical Risk Management

\section{Publish your work in this journal}

Therapeutics and Clinical Risk Management is an international, peerreviewed journal of clinical therapeutics and risk management, focusing on concise rapid reporting of clinical studies in all therapeutic areas outcomes, safety, and programs for the effective, safe, and sustained use of medicines. This journal is indexed on PubMed Central, CAS,

\section{Dovepress}

EMBase, Scopus and the Elsevier Bibliographic databases. The manuscript management system is completely online and includes a very quick and fair peer-review system, which is all easy to use. Visit http://www.dovepress.com/testimonials.php to read real quotes from published authors.

Submit your manuscript here: http://www.dovepress.com/therapeutics-and-clinical-risk-management-journal 